\title{
Implementation of IoT in Patient Health Monitoring and Healthcare for Hospitals
}

\author{
Liew Yi Kent ${ }^{1, *}$ Intan Farahana Binti Kamsin ${ }^{2}$ \\ ${ }^{1,2}$ Asia Pacific University of Technology and Innovation, Technology Park Malaysia, Bukit Jalil, Kuala Lumpur, \\ Malaysia. \\ *Corresponding author. Email:tp051829@mail.apu.edu.my
}

\begin{abstract}
Hospitals around the world are facing overcrowding issue especially during the Covid-19 pandemic. IoT devices can be implemented to improve the efficiency at hospitals and reduce risks of doctors getting infected. By implementing IoT, health monitoring can be done remotely, and healthcare provided to patients will be better and timelier. The method used to get the sample for the research is the Stratified sampling method and survey questionnaires will be distributed to collect data from them. A proposed system will then be made to check the feasibility and effectiveness of the system. In the future, the system should improve along with advancements in IoT so everyone will have ease of mind using the system. The aim of this research and proposal is to implement a solution for hospitals to improve health monitoring and provide better and timelier healthcare for patients.
\end{abstract}

Keywords: Healthcare, Health Monitoring, IoT, RFID, Wi-Fi, ZigBee.

\section{INTRODUCTION}

As healthcare became more and more available for everyone, the problem of overcrowding in hospitals became more apparent [1] [32, 33]. Ever since the Covid19 pandemic started in 2020, hospitals are also swarmed by patients infected by the virus and caused hospitals to not be able to care for other patients as hospitals are at full capacity [2, 3] [30] [34]. Medical staff will also face an increased risk of getting infected by the Covid-19 virus as they are in close contact with other infected patients [2] [23, 24, 25].

Other than the pandemic, [4] claimed that nurses spent the most time on medication related tasks, such as documentation and preparations as well as indirect patient care. The current process is not efficient as nurses need to manually check on the patient's status as well as administering medication for the patient when necessary [5]. That could waste the hospital's resources such as money and time which could be used to improve the care provided to patients $[4,5]$.

This research will propose an IoT based health monitoring and healthcare system using ZigBee, RFID and $\mathrm{Wi}-\mathrm{Fi}$ to improve efficiency and reduce excess miscellaneous work done by the medical staff. This will allow hospitals to care for more patients as well as remotely monitor a patient's condition even they are not present at the hospital which could reduce the risks of them getting infected by Covid-19. The medical staff can also access the history of the patient's condition as the data will be stored in the cloud where anyone with proper authority can access the data through a web interface. With the patient's history, the medical staff can also decide what will be the best treatment to be provided for the patient.

\section{RELATED WORK}

This section of the research will look at past research that are done which are related to the topic of this proposal. Studying past research will help identify flaws or drawbacks of current systems so improvements can be made in the new proposed system. Other than that, this section will compare several other similar existing systems. Some of the research domains that are reviewed for this research are:

$$
\begin{array}{ll}
\text { i. } & \text { Internet of Things (IoT) } \\
\text { ii. } & \text { Types of Patients } \\
\text { iii. } & \text { Health monitoring } \\
\text { iv. } & \text { Healthcare } \\
\text { v. } & \text { IoT Health Monitoring }
\end{array}
$$

\subsection{Internet of Things (IoT)}

There have been four phases of industrial revolution since the early $18^{\text {th }}$ century and the world is now in the 
fourth phase, which is Industry Revolution 4.0, otherwise known as the Digital Revolution [6]. According to [6, 7], the Industry Revolution 4.0 means to integrate complex machinery and devices with sensors and software through networks to generate information to make more informed decisions. In other words, the Industry Revolution 4.0 is driven by the Internet of Things, which allow connected devices to communicate with each other to capture data and convert to useful information [8].

To achieve that, IoT devices come equipped with sensors, actuators, and processors as well as transceivers for wireless communication [9] [27, 28]. Sensors and actuators are devices that interact with the physical environment to collect data, which are then processed to derive useful information [9] [29] [31]. For wireless communications, IoT can use different protocols to function which include Wi-Fi, Cellular, 6LoWPAN, ZigBee, RFID and NFC, where according to a system's requirements and limitations, will be using different protocols $[9,10]$.

\subsection{Types of Patients}

Hospitals need to admit many patients within a day and according to [1], emergency departments often face issues of overcrowding. As a result, a triage system is implemented to prioritise patients based on their symptoms [11, 12]. This issue became more apparent since the start of the Covid-19 pandemic where hospitals are overwhelmed with patients, so screening patients accurately to their respective urgency is very important $[11,12]$. Having said that, different patients would require different type of care provided by the hospital so they can be treated more effectively and efficiently [1].

\subsection{Health Monitoring}

Health monitoring means to monitor the health condition of an individual, which could measure various parameters such as heart rate, blood pressure, body temperature and respiration rate [13] [26]. Having the health of a patient constantly monitored means if the device detects any anomaly, it can instantly warn or notify a doctor for immediate treatment before the patient's condition would worsen [14]. Having a health monitoring system in a hospital can be advantageous as it can help detect illnesses early, monitor patients continuously as well as preventing illnesses from getting worse and reducing unnecessary deaths [13].

\subsection{Healthcare}

Healthcare is the service provided by hospitals or other medical facilities for patients, which is why hospitals should attempt to deliver good quality healthcare services for their patients [15]. According to $[15,16]$, some of the factors that affect the quality of healthcare services and should be improved are reliability, hygiene, equipment, adequate facilities, desire to serve and so on. On the other hand, reference [16] stated that interactions between medical staff and patients will impact the patient's satisfaction level on the healthcare provided. The healthcare provided to patients could also vary for different demographics, such as senior citizen needing more thorough care compared to other age groups [17].

\subsection{IoT Health Monitoring}

To implement IoT into health monitoring means to include the sensors that monitor vital signs into a network or the cloud so that health monitoring can be done anywhere and anytime [14]. Reference [18] stated that integrating health monitoring with IoT can improve efficiency of providing aid during an emergency while also improving the quality of life for patients. According to [19], using IoT also indicates that patient's health condition can be monitored and stored in the cloud which allows doctors to make better decisions based on the patient's specific situation.

\subsection{Similar Systems}

This section will discuss about other similar systems and these systems are focused on smart health monitoring and healthcare.

\subsubsection{System 1}

[14] proposed a framework for IoT health monitoring system that can detect a patient's heart rate, blood pressure, temperature, respiration rate, and activity which has a transmitter and a receiver section as shown in Figure 1. The sensors used are then connected to an Arduino Uno microcontroller which is being powered by an external power supply. It will be handling the data received from the sensors before sending the processed data to a web server via GSM [14]. After that, the received information can be represented as a graph at the webpage to display a patient's health condition [14]. With this proposed system, doctors, or other health workers can easily monitor their patients' status from anywhere and anytime as long as they have access to the Internet [14]. If there are anomalies in detected in the graphs, then immediately action can be taken to provide aid to the patient, so no precious time will be wasted before getting treatment for the patient while also avoiding the patient's condition from getting worse [14]. 


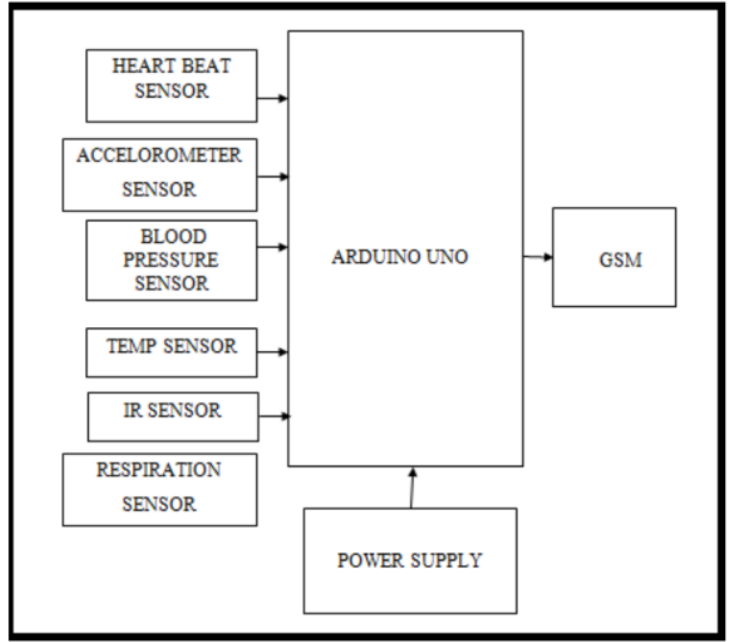

Figure 1 Block Diagram of Health Monitoring System [14]

\subsubsection{System 2}

Figure 2 below shows a health monitoring system proposed by [18] which can monitor a patient's blood pressure, heart rate and body temperature. The sensors are connected to a Raspberry Pi 3 microcomputer which is powered by a $5 \mathrm{~V}$ USB-B cable [18]. The data collected from the sensors are processed and can be sent to a mobile application or a web server using Bluetooth, Wi$\mathrm{Fi}$, or GSM. GSM is used to send the patient's vital signs to a phone via SMS while the Wi-Fi module is used to connect the system to a web application through the MQTT protocol. Other than that, the system uses Bluetooth for short range transmission so that patients can check their health on their phone through a mobile application.

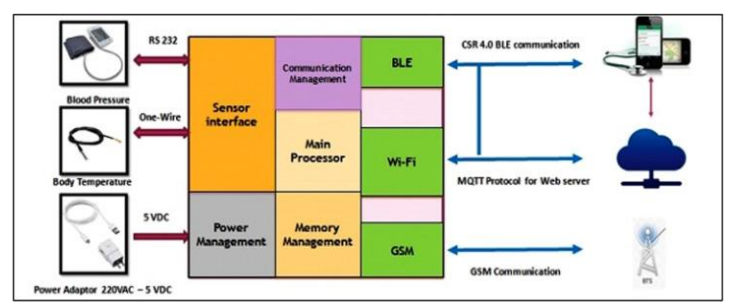

Figure 2 Functional Architecture of Health Monitoring System [18]

Table 1. Comparison Between Similar Systems

\begin{tabular}{|l|l|l|l|}
\hline & System 1 & System 2 & System 3 \\
\hline Monitoring Sensors & $\begin{array}{l}\text { Heartbeat, accelerometer } \\
\text { sensor, blood pressure, body } \\
\text { temperature, infrared, } \\
\text { respiration }\end{array}$ & $\begin{array}{l}\text { Blood pressure, heart rate, body } \\
\text { temperature }\end{array}$ & $\begin{array}{l}\text { Body temperature, heart rate, } \\
\text { room temperature, humidity, } \\
\text { gas }\end{array}$ \\
\hline Communication Protocols & GSM & GSM, BLE, Wi-Fi & Wi-Fi \\
\hline Processing Device & Arduino Uno & Raspberry Pi 3 & ESP32 \\
\hline Server/Database & Yes & Yes & Yes \\
\hline Alert & No & No & No \\
\hline Patient Tracking & Yes & No & No \\
\hline Remote Monitoring & Yes & Yes & No \\
\hline
\end{tabular}

\subsubsection{System 3}

System 3 is a Healthcare Monitoring System proposed by [19] which has three fundamental stages, sensors, data processing and web interface. This proposed system can detect a patient's body temperature, heart rate as well as the environment of the hospital such as temperature, humidity, and gas [19]. As shown in Figure 3, the sensors are connected to a ESP32 microcontroller that is connected to a battery power supply, which will be the centre of the system and collect data from the sensors before transferring them to a web server via its built-in Wi-Fi module [19]. If a medical staff wants to access the data on the web server, they can access it though a web interface on any device that can browse the Internet [19]. The web server will also be password protected to increase security, so the medical staff must enter the correct password. On the web interface, the data will be presented graphically so the medical staff can quickly and easily understand the patient's condition or diagnosing the patient [19].

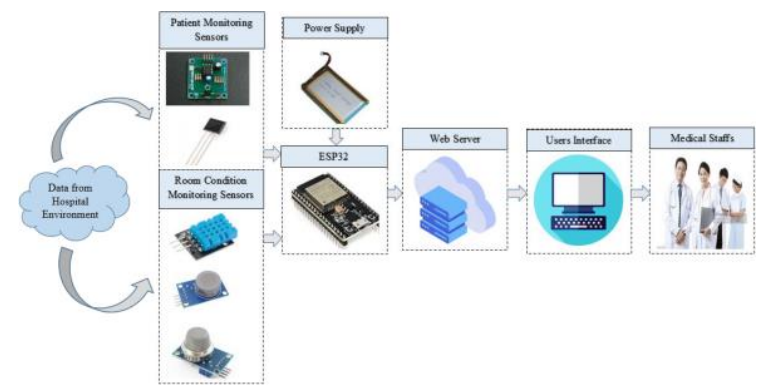

Figure 3 Overall System Architecture of Healthcare Monitoring System [19]

\subsubsection{Comparison of Similar Systems}


Table 1 below shows the comparison of features and components of three similar systems. For monitoring sensors, system 1 has the most sensors so it could monitor more vital signs to detect any changes on a patient's health condition, while system 3 also offers monitoring for the hospital environment so it gives more context to the data received about the patient. System 2 uses multiple communication protocols for different situations while the other two only uses one, which is sufficient for their use cases. All three systems use a different processing device, but if the system is very demanding then the Raspberry Pi 3 in system 2 will be better suited. These 3 systems also include a server or database to store data collected from the sensors, so that medical staff can check on the patient's history files to gain more context into the patient's health condition [20]. Other than that, system 1 meets most of the criteria, a server/database, an alerting feature, patient tracking function as well as remote monitoring function. The proposed system will include multiple monitoring sensors, uses multiple communication protocols, has a server, alert, patient tracking, remote monitoring with additional features like automated drug dispenser and equipment tracking [5].

\subsection{Overview of Proposed System}

The proposed system can monitor remote patients as well as normal patients. For remote patients, they will wear a health monitor and an activity monitor to monitor their health condition and activity which will send the data to the hospital's cloud server through Wi-Fi or cellular signal. For normal patients, they will have sensors attached to them for monitoring heart rate, blood pressure, respiration rate, oxygen level and body temperature. The data will be transmitted to the local database that is connected to the cloud through Wi-Fi and ZigBee which allows for real-time monitoring by the medical staff from anywhere with Internet access. The database will also allow for checking a patient's medical history. If there any anomalies in a patient's condition, the system would detect it and immediately notify and alert the medical staff, so immediate treatment can be given or having an ambulance sent for the remote patient. The medical staff can also schedule a time for medicine to be automatically dispensed to a patient via IV drops. Other than that, medical equipment will have an RFID tag for location tracking by RFID readers all over the hospital, so no time will be wasted looking for equipment that is misplaced. The process of the system is shown in Figure 4 below.

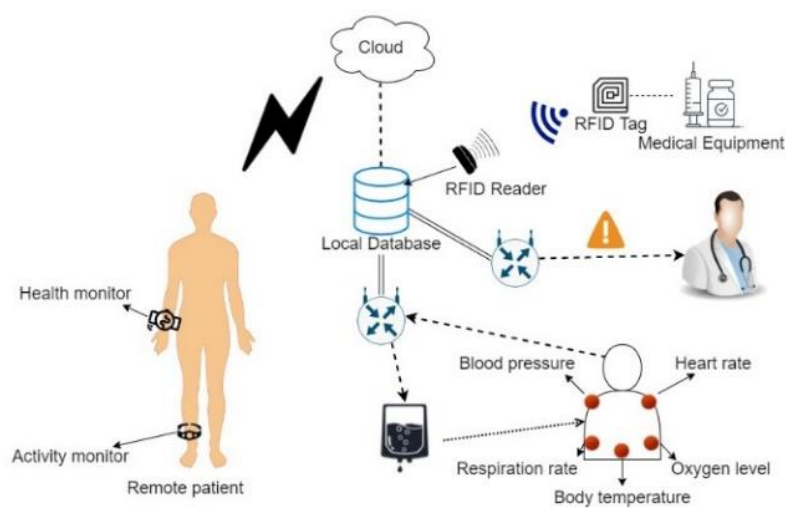

Figure 4 Overview of the Proposed System

\section{RESEARCH METHODOLOGY}

This research will be using the quantitative research approach to study the factors that cause inefficiencies in current systems. The sample size of respondents is 25 which are 15 from hospital patients and 10 from medical staff. The sampling method used to identify the sample is stratified sampling which is a probability sampling method. This method is used because the sample is divided into two subgroups which are the patients and medical staff, this way the data collected will be more precise. The respondents are the patients and medical staff because they are the most involved with the health monitoring and healthcare systems.

A survey questionnaire will be distributed to the respondents to collect data for the research which includes some multiple-choice questions and questions with 7-point Likert scale about the current systems. A mix of open and closed questions will be used for the questionnaire which are objective and Likert style questions. After collecting the data, it will be analysed to summarise what the respondents think of the current systems.

\section{DISCUSSION}

This section of the article will discuss about the significance of this research. This research is important especially during the Covid-19 pandemic where hospitals are flooded with patients infected by Covid-19. This situation only worsens the issue of hospital overcrowding as other patients will not receive any medical care as the hospital cannot spare more resources for them. If the proposed system is implemented, it could reduce the workload of medical staff and make more efficient use hospital resources which allows hospitals to aid more patients. It could also provide automated basic medicine administering so that patients with lower urgency will still get treated even while staff are busy. This research will help discover how IoT can help in innovating and changing the medical sector in the future. 


\section{CONCLUSION}

This research proposed an IoT based health monitoring and healthcare system for hospitals using RFID, ZigBee, and Wi-Fi to make processes in the hospital more efficient and reduce workload of medical staff. It can reduce the time for physical contacts between medical staff and patients as monitoring can be done from anywhere. Time wasted locating medical equipment will also be reduced as their location will be tracked by RFID. As IoT improves in the future, data transmission will be faster, more reliable, and robust while security also improves on every device on the IoT network so that everyone can feel more reassured about their privacy.

While IoT is still a relatively new technology that is still developing, it has a few limitations, namely security. With more IoT devices on the network, it also means that there are more nodes that a hacker or any person with an ill intent can get access to [21]. Since every device on the network could be made by different vendors, the security protocol implemented in each of them could be different while the hacker can easily find and exploit the device with the weakest security [21]. In addition to security, the privacy of the patients is also a notable concern, as the system will store a big amount data about them [21, 22]. Some patients might value their privacy and disagree getting a better treatment from the hospital. Other than that, if the data stored on the web server is stolen by a hacker, someone will be held accountable for the incident, whether it be the IT security, hospital management or even the device vendors. Future works should improve on the two points mentioned above, security and privacy, which go hand-in-hand. If security gets better, the privacy of patients will be better, and more people will start trusting in the system as well.

\section{ACKNOWLEDGMENT}

Highest appreciation to Asia Pacific University, Malaysia for the opportunity on the article publication.

\section{REFERENCES}

[1] Zachariasse J. M., Hagen, V. V. D., Seiger, N., Mackway-Jones, K., Veen, M. V. and Moll, H. A. Performance of Triage Systems in Emergency Care: A Systematic Review and Meta-analyis. BMJ Open. 2019(9), 2019.2 DOI: http://dx.doi.org/10.1136/bmjopen-2018-026471

[2] Bielicki, J. A., Duval, X., Gobat, N., Goossens, H., Koopmans, M., Tacconelli, E. and Werf, S. V. D. Monitoring Approaches for Health-care Workers During the COVID-19 Pandemic. THE LANCET Infectious Diseases. 20(10), 2020, pp. 1101-1216.
DOI: 3099(20)30458-8

[3] Tan, C. S., Lokman, S., Rao, Y., Kok, S. H. and Ming, L. C. Public and Private Sectors Collective Response to Combat Co-vid-19 in Malaysia. Journal of Pharmaceutical Policy and Practice. 14:40, 2021. DOI: https://doi.org/10.1186/s40545-021-00322-X

[4] Glantz, A., Örmon, K. and Sandström, B. "How Do We Use the Time?" - An Observational Study Measuring the Task Time Distribution of Nurses in Psychiatric Care. BMC Nursing. 18:67, 2019. DOI: https://doi.org/10.1186/s12912-019-0386-3

[5] Joseph, S., Francis, N., John, A., Farha, B. and Baby, A. Intravenous Drip Monitoring System for Smart Hospital Using IoT. 2019 2nd International Conference on Intelligent Computing, Instrumentation and Control Technologies (ICICICT). 2019, pp. 835-839. DOI: https://doi.org/10.1109/ICICICT46008.2019.89932 41

[6] Dogaru, L. The Main Goals of the Fourth Industrial Revolution. Renewable Energy Perspective. Procedia Manufacturing. 46, 2020, pp. 397-401. DOI: https://doi.org/10.1016/j.promfg.2020.03.058

[7] Leong, W. D., Teng, S. Y., How, B. S., Ngan, S. L., Rahman, A. A., Tan, C. P., Ponnambalam, S. G., Lam, H. L. Enhancing the Adaptability: Lean and Green Strategy towards the Industry Revolution 4.0. Journal of Cleaner Production, 122870, 2020. DOI: https://doi.org/10.1016/j.jclepro.2020.122870

[8] Al-Ogaili, A. S., Alhasan, A., Ramasamy, A., Marsadek, M., Hashim, T. J. T., Al-Sharaa, A., Aadam, M. and Audah, L. IoT Technologies for Tackling COVID-19 in Malaysia and Worldwide: Challenges, Recommendations, and Proposed Framework. Computers, Materials \& Continua. 66(2), 2020, pp. 2141-2164. DOI: http://dx.doi.org/10.32604/cmc.2020.013440

[9] Moraes, T., Nogueira, B., Lira, V. and Tavares, E. Performance Comparison of IoT Communication Protocols. 2019 IEEE International Conference on Systems, Man and Cybernetics (SMC). Bari, Italy. 6-9 October 2019.2 DOI: https://doi.org/10.1109/SMC.2019.8914552

[10] Shafique, K., Khawaja, B. A., Sabir, F., Qazi, S. and Mustaqim, M. Internet of Things (IoT) for NextGeneration Smart Systems: A Review of Current Challenges, Future Trends and Prospects for Emerging 5G-IoT Scenarios. IEEE Access. 8, 2020, pp. 23022-23040. DOI: https://doi.org/10.1109/ACCESS.2020.2970118 
[11] Truog, R. D., Mitchell, C. and Daley, G. Q. The Toughest Triage - Allocating Ventilators in a Pandemic. New England Journal of Medicine. 382(21), 2020, pp. 1973-1975. DOI: https://doi.org/10.1056/NEJMp2005689

[12] Wang, Q. The Role of Triage in the Prevention and Control of COVID-19. Infection Control \& Hospital Epidemiology. 2020(41), 2020, pp. 772-776. DOI: https://doi.org/10.1017/ice.2020.185

[13] Malasinghe, L. P. Ramzan, M. and Dahal, K., Remote Patient Monitoring: A Comprehensive Study. Journal of Ambient Intelligence and Humanized Computing. 10, 2019 pp. 57-76. DOI: https://doi.org/10.1007/s12652-017-0598-X

[14] Sam, D., Srinidhi, S., Niveditha, R., Amudha, S. and Usha, D. Progressed IOT Based Remote Health Monitoring System. International Journal of Control and Automation. 13(2s), 2020, pp. 268-273.

[15] Asnawi, A. A., Awang, Z., Afthanorhan, A., Mohamad, M. and Karim, F. The Influence of Hospital Image and Service Quality on Patients' Satisfaction and Loyalty. Management Science Letters. 9(6), 2019, pp. 911-921. DOI: http://dx.doi.org/10.5267/j.msl.2019.2.011

[16] Babroudi, N. E. P., Sabri-Laghaie, K., Ghoushchi, N. G. Re-evaluation of the Healthcare Service Quality Criteria for the Covid-19 Pandemic: Znumber Fuzzy Cognitive Map. Applied Soft Computing. 112, 2021, DOI: https://doi.org/10.1016/j.asoc.2021.107775

[17] Andersen, Y. M. F., Egeberg, A., Skov, L. and Thyssen, J. P. Demograohics, Healthcare Utilization and Drug Use in Children and Adults with Atopic Dermatitis in Denmark: A Population-based Crosssectional Study. Journal of the European Academy of Dermatology and Venereology. 33, 2019, pp. 1133-1142. DOI: https://doi.org/10.1111/jdv.15424

[18] Swaroop, K. N., Chandu, K., Gorrepotu, R. and Deb, S. A Health Monitoring System for Vital Signs Using IoT. Internet of Things. 5, 2019, pp. 116-129. DOI: https://doi.org/10.1016/j.iot.2019.01.004

[19] Islam, M.M., Rahaman, A. and Islam, M.R. Development of Smart Healthcare Monitoring System in IoT Environment. SN Computer Science. 1:185, 2020, DOI: https://doi.org/10.1007/s42979020-00195-y

[20] Lucarotti, P. and Burke, F. Patient History as a Predictor of Future Treatment Need? Considerations From a Dataset Containing Over Nine Million Courses of Treatment. British Dental
Journal. 228, 2020, pp. 345-350. DOI: https://doi.org/10.1038/s41415-020-1305-4

[21] Tawalbeh, L., Muheidat, F., Tawalbeh, M. and Quwaider, M., IoT Privacy and Security: Challenges and Solutions. Applied Sciences. 10(12):4102, 2020, DOI: http://dx.doi.org/10.3390/app10124102

[22] Menard, P. and Bott, G. J., Analyzing IOT Users' Mobile Privacy Concers: Extracting Privacy Permissions Using a Disclosure Experiment. Computers \& Security. 95:101856, 2020, DOI: https://doi.org/10.1016/j.cose.2020.101856

[23] Poongodi, M., Nguyen, T.N., Hamdi, M. and Cengiz, K., 2021. A Measurement Approach Using Smart-IoT Based Architecture for Detecting the COVID-19. Neural Processing Letters, pp.1-15.

[24] L. Tan, K. Yu, A. K. Bashir, X. Cheng, F. Ming, L. Zhao, X. Zhou, "Towards Real-time and Efficient Cardiovascular Monitoring for COVID-19 Patients by 5G-Enabled Wearable Medical Devices: A Deep Learning Approach", Neural Computing and Applications, 2021, https://doi.org/10.1007/s00521021-06219-9.

[25] K. Yu, L. Tan, X. Shang, J. Huang, G. Srivastava and P. Chatterjee, "Efficient and Privacy-Preserving Medical Research Support Platform Against COVID-19: A Blockchain-Based Approach”, IEEE Consumer Electronics Magazine, doi: 10.1109/MCE.2020.3035520.

[26] H. Li, K. Yu, B. Liu, C. Feng, Z. Qin and G. Srivastava, "An Efficient Ciphertext-Policy Weighted Attribute-Based Encryption for the Internet of Health Things," IEEE Journal of Biomedical and Health Informatics, 2021, doi: 10.1109/JBHI.2021.3075995.

[27] Y. Gong, L. Zhang, R. Liu, K. Yu and G. Srivastava, "Nonlinear MIMO for Industrial Internet of Things in Cyber-Physical Systems," IEEE Transactions on Industrial Informatics, vol. 17, no. 8, pp. 5533-5541, Aug. 2021, doi: 10.1109/TII.2020.3024631.

[28] So-In, C., 2020. Efficient SDN-Based Traffic Monitoring in IoT Networks with Double Deep QNetwork. In Computational Data and Social Networks: 9th International Conference, CSoNet 2020, Dallas, TX, USA, December 11-13, 2020, Proceedings (Vol. 12575, p. 26). Springer Nature.

[29] Tran, D.N., Nguyen, T.N., Khanh, P.C.P. and Trana, D.T., 2021. An iot-based design using accelerometers in animal behavior recognition systems. IEEE Sensors Journal.

[30] Subramani, P., Srinivas, K., Sujatha, R. and Parameshachari, B.D., 2021. Prediction of muscular 
paralysis disease based on hybrid feature extraction with machine learning technique for COVID-19 and post-COVID-19 patients. Personal and Ubiquitous Computing, pp.1-14.

[31] Begum, S., Banu, R., Ahamed, A. and Parameshachari, B.D., 2016, December. A comparative study on improving the performance of solar power plants through IOT and predictive data analytics. In 2016 International Conference on Electrical, Electronics, Communication, Computer and Optimization Techniques (ICEECCOT) (pp. 89-91). IEEE.

[32] Deepa J, Ranjini, Sharanya Raj, Dr. Parameshachari B D, 2018, Soldier Health and Position Tracking System using GPS and GSM Modem., INTERNATIONAL JOURNAL OF ENGINEERING RESEARCH \& TECHNOLOGY (IJERT) NCESC - 2018 (Volume 6 - Issue 13).

[33] Subramani, P., Al-Turjman, F., Kumar, R., Kannan, A. and Loganthan, A., 2021. Improving medical communication process using recurrent networks and wearable antenna s11 variation with harmonic suppressions. Personal and Ubiquitous Computing, pp.1-13.

[34] Prabu, S., Velan, B., Jayasudha, F.V., Visu, P. and Janarthanan, K., 2020. Mobile technologies for contact tracing and prevention of COVID-19 positive cases: a cross-sectional study. International Journal of Pervasive Computing and Communications. 